\title{
Electrostatic Contributions to Carcinogenesis
}

\author{
L. John Gagliardi' ${ }^{1}$, Daniel H. Shain ${ }^{2 *}$ \\ ${ }^{1}$ Departments of Physics, Rutgers The State University of New Jersey, Camden, NJ, USA \\ ${ }^{2}$ Departments of Biology, Rutgers The State University of New Jersey, Camden, NJ, USA \\ Email: gagliard@scarletmail.rutgers.edu, ${ }^{\star}$ dshain@camden.rutgers.edu
}

How to cite this paper: Gagliardi, L.J. and Shain, D.H. (2020) Electrostatic Contributions to Carcinogenesis. Open Journal of Biophysics, 10, 27-45. https://doi.org/10.4236/ojbiphy.2020.10100 $\underline{3}$

Received: November 29, 2019

Accepted: January 13, 2020

Published: January 16, 2020

Copyright $\odot 2020$ by author(s) and Scientific Research Publishing Inc. This work is licensed under the Creative Commons Attribution International License (CC BY 4.0).

http://creativecommons.org/licenses/by/4.0/

\section{(c) (i) Open Access}

\begin{abstract}
Nanoscale electrostatics plays important roles in aster (spindle) assembly and motion, nuclear envelope breakdown and reassembly, and in force generation at kinetochores, poles, and chromosome arms for prometaphase, metaphase, and anaphase-A chromosome motions during mitosis. A large body of experimental evidence also suggests a role for electrostatics as the trigger for mitosis, which is considered here particularly in the context of cancer. Cancer cells are characterized by impaired intercellular electrical communication and adhesive contact as well as a loss of contact inhibition, conditions associated with increased cell surface negativity relative to their normal counterparts. Dividing cells have also been associated with lower transmembrane potentials and altered intracellular ionic concentrations. Here we propose that cancer cells are distinguished by abnormal trans- and intramembrane electric potentials, leading to the loss of active $\mathrm{Na}+\mathrm{K}+$ plasma membrane pumping, increased intracellular concentrations of sodium and other ions, and alkaline nucleo-cytoplasmic $\mathrm{pH}$, all of which are associated with and integral to carcinogenesis.
\end{abstract}

\section{Keywords}

Division, Malignant, Mitosis, Cancer, Alkalinity

\section{Introduction}

The electromagnetic interaction is primarily responsible for the structure of matter from atoms to objects. Much of physics, all of chemistry, and most of biology are in this size realm. Primitive eukaryotic cells had to divide prior to the evolution of many biological mechanisms, and it is reasonable to assume that electrostatics, a component of the electromagnetic interaction, played (and continues to play), an important role in the mechanics of chromosome motions during mitosis [1] [2] [3] [4] [5]. 
The cancer problem is characterized by the existence of cells that divide when they should not. An explanation of this behavior has emerged as one of the signature problems in biology. With an abundance of proposals regarding the trigger for cancerous cell division, how does one decide which approach is the most compelling? Regarding scientific models, the renowned physicist Paul Ehrenfest suggested that they should be framed in such a manner that "the essence lies in recognizing the connections in all directions." Recognizing a number of connections between increased cancer cell surface negativity motivated Van Beek et al. [6] to suggest some time ago that increased cell surface membrane-bound negative charge is a general feature of cancer cells.

Mammalian cells are known to have bound negative charges on their surfaces. This surface charge density $\sigma \mathrm{C} / \mathrm{m}^{2}$ (Coulombs per square meter) is due largely to ionized carboxyl groups of sialic acid residues [7] [8] [9]. Net surface negativity is primarily a function of the density of these anionic groups anchored in the membrane at the cell surface, but it also depends on the $\mathrm{pH}$ and ionic strength of the surrounding medium [10]. Other acidic groups may reside at the cell surface [11], and some cells have anionic groups associated with RNA at their periphery [12].

Studies on cells transformed by oncogenic viruses suggest that the altered growth pattern of these cells is caused by changes at the cell surface [13]. Importantly, changes at the cell surface often involve the carbohydrate components of glycolipids and glycoproteins, including an increase of fucose-labeled glycoprotein with increased sialic acid density [6] [11] [14] [15]. Quite some time ago, it was proposed that increased sialic acid density at the surfaces of transformed and malignant cells could be a general characteristic of cancer cells [6].

It is well established that transformed and malignant cells suffer from drastically impaired intercellular communication [16]. One of the most well known characteristics of cancer cells is that, unlike normal cells, they do not stop dividing upon functional cellular contact (i.e., cellular contact that leads to the expression of a signal inhibitory to S-phase initiation). Studies on a variety of mature cell types in vivo have shown that the great majority are arrested in the $\mathrm{G}_{1}$ period of the cell cycle [17], and must pass through the S period of DNA synthesis before entering mitosis, although a small fraction may be arrested in the $\mathrm{G}_{2}$ period [18].

A number of studies have demonstrated the importance of sialic acid [11] [19] [20]-[25] and other cell surface carbohydrates [11] [26] in cell contact and recognition phenomena. In principle, increased density of sialic acid could prevent the intimate association necessary to establish intercellular contact, either directly due to the increased charge, or indirectly by masking other carbohydrate residues involved in functional contact.

Another long-standing set of observations concerns differences between the transmembrane electric potential of cycling (i.e., dividing) and non-cycling cells [27] [28] [29] [30]. These studies have shown that the former have much lower 
transmembrane potentials and different intracellular concentrations of inorganic ions (henceforth simply designated "ions"). An example of a significant decrease in transmembrane potential $\Delta V_{m}$ is observed in cellular adaptation from in vivo non-dividing conditions to growth (dividing cells) in vitro. Thus the interphase $\left(G_{1}\right) \Delta V_{m}$ level of -50 to $-60 \mathrm{mV}$ for typical mature somatic cells in vivo undergoes a change to in vitro $G_{1}$ levels in the vicinity of $-10 \mathrm{mV}$. This well-known decrease appears to be a general phenomenon. Also noteworthy is the pronounced membrane depolarization (approx. $-90 \mathrm{mV}$ to $-10 \mathrm{mV}$ ) accompanying malignant transformation of somatic cells in vivo [31] [32] [33].

We propose here that electrostatics was, and currently remains, integral to the initiation of cell division (i.e., mitogenesis). Moreover, differences in ion concentrations and overall ionic strength are both important aspects of the disparity in measured transmembrane potentials of dividing versus non-dividing cells, as considered below.

\section{Mechanical Equilibrium of a Membrane}

The mechanical equilibrium of a model half-membrane is depicted schematically in Figure 1. The hemispherical shell, representing half of the membrane, is in equilibrium under the action of a uniform surface tension acting in the $-x$ direction from the rest of the membrane, and surface forces acting perpendicularly outward from the surface everywhere over the hemisphere. The surface forces arise from both the pressure difference across the membrane and a surface electrical force per unit area of the membrane (i.e., membrane electrostatic stress). These forces may be envisaged if one considers a charged balloon in equilibrium under 1) the surface tension forces due to the elastic deformation of the rubber, 2) the pressure difference between the inside and outside of the balloon, and 3) a membrane electrostatic stress (ES) due to the mutual repulsion of like negative

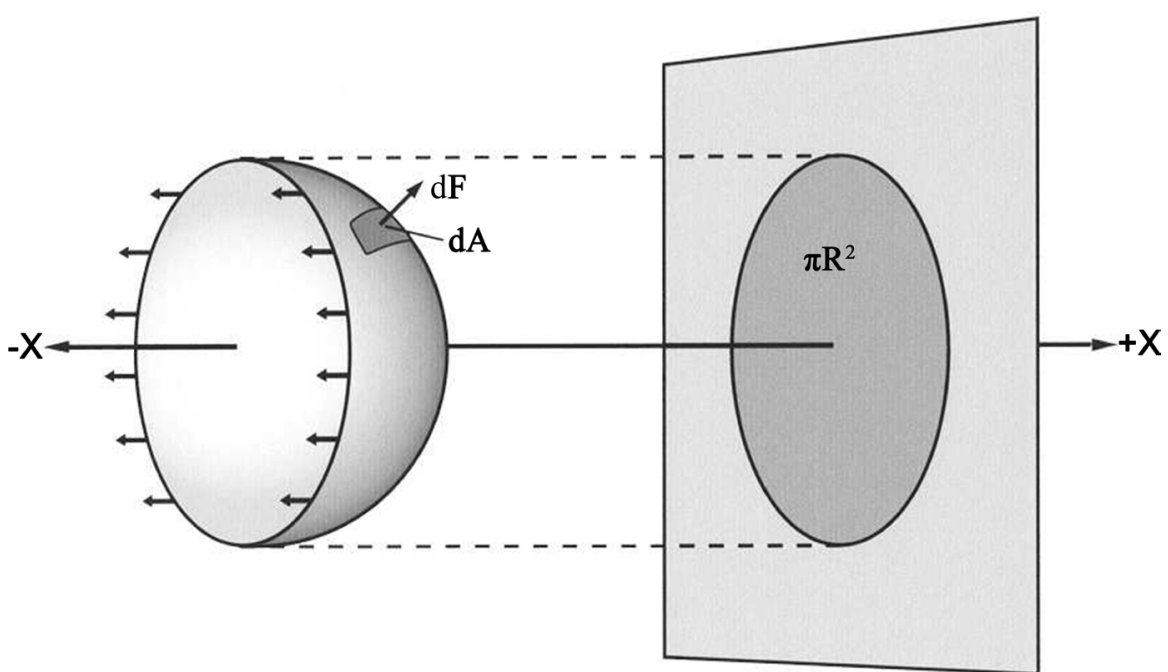

Figure 1. Model half-membrane in equilibrium. The spherical half-membrane is in equilibrium under membrane surface tension forces acting to the left and electrical stress plus hydrostatic force components acting to the right. 
charges fixed to the surface. The use of a spherical shell is clearly an idealization; however, the analysis presented here is valid for a large class of ellipsoids of revolution, with the mathematics being far simpler for a spherical geometry.

The differential electrostatic force $\mathrm{d} F_{e}$ acting perpendicularly to an element of area $\mathrm{d} A$ of a charged surface can be expressed by the following equation [34].

$$
\mathrm{d} F_{e}=\left(\varepsilon E^{2} / 2\right) \mathrm{d} A=\left(\sigma^{2} / 2 \varepsilon\right) \mathrm{d} A
$$

where $E^{2}$ is the magnitude squared of the electric field at $\mathrm{d} A, \varepsilon$ is the local permittivity just outside the surface at $\mathrm{d} A$, and $\sigma$ is the net surface charge per unit area. The half-membrane will be in equilibrium if the surface tension element of force, $\gamma \mathrm{d} l$ (where $\gamma$ is the membrane surface tension in $\mathrm{N} / \mathrm{m}$ ) integrated around the circumference of the membrane $(2 \pi R \gamma)$, is equal to the total force in the $+x$ direction. Integrating the component in the $+x$ direction of the total differential force $\mathrm{d} F$, which arises from ES plus the pressure difference $\left(p_{1}-p_{2}\right)$ across the membranes, we find that

$$
2 \gamma / R=\Delta p+\sigma^{2} / 2 \varepsilon
$$

where $\Delta p=\left(p_{1}-p_{2}\right)$, where $p_{1}$ is the pressure inside. This equation governs the equilibrium of the model half-membrane.

Studies have shown that ES manifests itself in membrane equilibrium. After a series of experiments on cell deformability, Weiss [35] concluded that terminal sialic acids contribute to the mechanical properties of the cell periphery through electrostatic repulsion between their own and other anionic groups. Cells in this study became more deformable after incubation with neuraminidase, an enzyme which removes negatively charged sialic acid residues from the cell surface. In animal cells, the terminal sialic acids are attached to cell (plasma) membrane proteins, which are firmly anchored in the lipid bilayer. Therefore, it is reasonable to assume that the repulsive interactions between these groups are primarily responsible for the observed decrease in the deformability of cells with more surface negative charge.

If $\Delta p$ were larger than $\sigma^{2} / 2 \varepsilon$, removal of a significant portion of surface charge by treatment with neuraminidase would not be consistent with the observed increase in the deformability of these cells [35]. We may therefore assume that the $\Delta p$ term is at most comparable in magnitude to the $\sigma^{2} / 2 \varepsilon$ contribution to membrane stress. Accordingly, it is possible to obtain a good approximation for the equilibrium of a membrane from (2) written as:

$$
\sigma^{2} / 2 \varepsilon \approx 2 \gamma / R
$$

It is well established in electrochemistry [36] that the permittivity of the first few water layers outside a charged surface is an order of magnitude smaller than that of the bulk phase. The effective permittivity of water as a function of distance from a charged surface has been determined by atomic force microscopy [37] to increase monotonically from $4-6 \varepsilon_{0}$ at the interface to $78 \varepsilon_{0}$ at a distance of $25 \mathrm{~nm}$ from the interface. The values of dielectric constants $k(x)$ at distances of 1,2, 3 and $4 \mathrm{~nm}$ from a charged surface were measured to be $9,21,40$ and 60 , 
respectively. The value of $\varepsilon$ at the membrane outer (cytoplasmic) surface may therefore be conservatively estimated as $30 \mathcal{E}_{0}$, where $\varepsilon_{0}$ is the permittivity of free space, $8.85 \mathrm{pF} / \mathrm{m}$ (picoFarads/meter). The experiment was carried out with mica, which has a surface charge density that varies from 1 to $50 \mathrm{mC} / \mathrm{m}^{2}$, in the same range as biological surfaces [38] [39].

The range for $\gamma$ may be taken as 0.1 to 1.0 dyne/cm $(0.1$ to $1 \mathrm{mN} / \mathrm{m})$ [40]. These values, when substituted into (3), using $R=20 \mu \mathrm{m}$ and $\varepsilon=30 \varepsilon_{0}=270$ $\mathrm{pF} / \mathrm{m}$ (picoFarads/meter), give a range of values for $\sigma$ from 73 to $230 \mu \mathrm{C} / \mathrm{m}^{2}$ (microCoulombs/square meter). Values of $\sigma$ in this range are sufficient to exert an electrostatic stress comparable to $2 \gamma / R$ in the cell membrane. Experimental values for the surface charge density of biological and artificial lipid membranes range from 0.4 to $160 \mathrm{mC} / \mathrm{m}^{2}$ (milliCoulombs/square meter) [38] [39] [41] [42]. This calculation strongly suggests that electrostatic stress is an important factor in the equilibrium and thermodynamics of the plasma membrane.

Cell electrophoresis studies have identified a $12 \%$ - $18 \%$ increase in plasma membrane negativity just prior to cell division [43] [44], consistent with an observed increase of $50 \%$ in the whole cell content of sialic acid during mitosis [45]. Importantly, note that

$$
\Delta\left(\sigma^{2}\right) / \sigma^{2} \approx 2 \Delta \sigma / \sigma
$$

and an increase of $15 \%$ in cell membrane negativity corresponds to a $30 \%$ increase in cell surface electrostatic stress. Similar increases may be expected for the membranes of the nuclear envelope [2] and mitochondria.

Increased ES on the plasma membrane could explain the well-known observation that cells typically assume a more spherical shape (i.e., "round out") during cell division. As referenced above, an increased density of sialic acid in the surface glycoproteins of transformed and malignant cells has been observed in a number of studies. This is consistent with the common observation that cancer cells are characteristically rounded and "puffy" in appearance, possibly from permanently increased ES on these cells.

A number of experimental studies have revealed that the plasma membrane transport properties of transformed cells differ from their normal counterparts [46]. As indicated above, an increased membrane charge density, with its associated increase in ES, is large enough to be manifested in the equilibrium of cellular membranes. Increased ES, along with other factors, could therefore be related to the observed altered transport properties of the plasma membrane (see below).

Elevated surface charge may also interfere with the formation of gap junctions, which provide an important mechanism for coordinating the activities of neighboring cells. These specialized cell-cell junctions form between closely apposed cell membranes to directly connect the cytosols of the joined cells with narrow canals through which small molecules and ions can pass. A number of studies have shown that embryonic cells make and break gap junction connections in definite patterns, suggesting they play a role in coordinating cell division 
and growth [29] [47]. It therefore seems likely that the greatly increased intercellular electrical resistance of abnormal cells [16] is due to defective or reduced numbers of gap junctions, and that increased sialyl extension [6] from the greater surface charge density is at least partially responsible for this condition.

Thus abnormal cells exhibit a greatly increased intercellular electrical resistance, indicative of abnormal barriers to the flow of ions between cells. The greater surface charge exhibited by cancer cells has been associated with defective cell-cell contacts, likely a major contributing factor to the inability of cancer cells to form normal gap junctions. Because intercellular communication via properly functioning gap junctions is necessary, maintenance of non-mitogenic intracellular ionic concentrations over organ-size volumes may operate as a social control over cell division that is utilized by multicellular organisms (see below). This could be one possible explanation for the existence of local pockets of cancer cells-presumably with defective gap junctions and a resulting defective intercellular communication-within organs surrounded by normal cells, a common observation in tumor histology.

As noted, numerous observations regarding cancer cells have revealed that sialic acid is involved in cell contact [19] [23] [24] [26] [48] [49] [50] and growth control by functional contact [51] [52] [53]. For these and other reasons, an increased density of membrane-bound, negatively charged sialic acid at the cancer cell surface has been suggested as a general characteristic of malignant cells [6]. In the context of electrostatics, these experimental observations underlie the primary conditions that regulate mitogenesis. In particular, we propose that increased cell surface charge significantly influences the intramembrane electric potential, with implications for the active transport of ions as well as the operation of ion channels, as detailed below.

\section{Membrane Electric Potentials and Intracellular Ionic Concentrations}

Membrane potentials for biological cells are usually treated using the diffusion potential approach [54] [55], where it is assumed that the observed transmembrane potential (often designated as "membrane potential") is equal to the thermodynamic diffusion potential. However, an undesirable outcome of this approach is the emergence of negative permeability coefficients, which are unrealistic [56]. Ohki [57] [58], and MacDonald and Bangham [59] were the first to include the contribution of surface potentials to the observed transmembrane potential of biomembranes. Ohki presented experimental observations, accompanied by theoretical calculations, in seeking to clarify the relation between surface potentials, diffusion potential and transmembrane potential [56]. Surface potentials arise from the presence of charged groups at the outer (extracellular medium) and inner (cytoplasmic) surfaces of the plasma membrane. As described above, the negative charge at the outer cell membrane surface is primarily due to membrane-bound, ionized carboxyl groups of sialic acid residues. 
Consistent with the above discussions, we focus our attention on the outer cell surface charge and its resultant electric potential.

The characteristic Debye length of surface potentials for cells is of the order of $1 \mathrm{~nm}[60]$, so there is no possibility of resolving surface potentials from diffusion potentials. The surface potential at the inner (cytoplasmic) surface of a membrane is also primarily due to anionic groups [56] [61]. Additionally, polarization potentials $V_{p}$ from polarized molecules at the inner and outer surfaces of the membrane arise from surface molecules possessing dipole moments. It will be assumed here that the polarization potentials at membrane surfaces effectively cancel each other [56]. Thus, for the measured transmembrane potential, $\Delta V_{m}$, we have

$$
\Delta V_{m}=\varphi_{i}+\varphi_{o}+V_{d}
$$

where $V_{d}$ is (if $\varphi_{i}$ and $\varphi_{o}$ are 0 ) the diffusion potential arising from unequal ionic concentrations between the inside and outside of a cell, and $\varphi_{i}$ and $\varphi_{o}$ are the surface potentials at the cytoplasmic (inner), and extracellular (outer) cell membrane surfaces, respectively. Because of the surface electric potentials, the actual potential associated with the passive diffusion of ions is $\Delta V_{m}$, not $V_{d}$. To simplify, $V_{d}$ will be designated as the intramembrane potential in the presence of surface potentials, and as the diffusion potential in the absence of surface potentials; both are intramembrane electric potentials. Due to the increased outer surface membrane-bound negative charge described above, a larger negative surface potential (Figure 2) will exist at the outer surface of cancer cells.

The Nernst potential $V_{N}$ [62] of an ion in pure passive electrodiffusive equilibrium (no ion pumps) across a cell membrane is given by

$$
V_{N}=-(k T / Z e) \ln \left(C_{i} / C_{o}\right)
$$

where $C_{i} / C_{o}$ is the ratio of the ion's concentration inside vs. outside the cell, $Z$ is the valence of the ion, $e$ is the magnitude of the charge on an electron, $k$ is

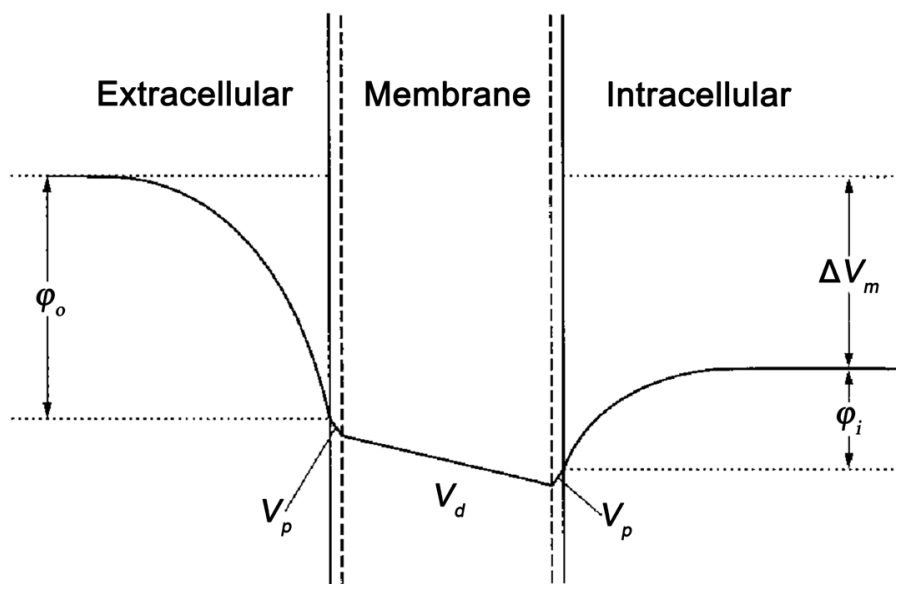

Figure 2. Schematic transmembrane electric potential profile. Surface potentials $\varphi_{i}$ and $\varphi_{o}$ are shown in relation to the intramembrane potential $V_{d}$. The polarization potentials $V_{p}$ and the measured trans-membrane potential $\Delta V_{m}$ are also depicted as described in the text. 
Boltzmann's constant, and $T$ is absolute temperature. The Nernst potentials of each of the ions in passive electrodiffusive equilibrium must be equal.

For a given potential difference $\left(C_{i} / C_{o}\right)^{1 / Z}$ is the same for all ions. Thus, for monovalent cations and anions $(Z= \pm 1)$, we have

$$
C_{i(+)} / C_{o(+)}=C_{o(-)} / C_{i(-)}
$$

where $C_{i(-)}$ and $C_{i(+)}$ are the intracellular concentrations of negatively and positively charged permeant ions, respectively, with a similar notation for extracellular ions. Concentrations of ions in the extracellular medium and the total concentration of intracellular impermeant ions $C_{n p}$, along with (7) and the bulk intracellular electroneutrality condition are sufficient to determine the concentrations of permeant ions in the cell in the absence of active transport.

A considerable body of research identifies a relationship among intracellular ionic concentrations, cell membrane electric potential and mitogenesis. Mitotically active cells are often found to have low $(-10$ to $-20 \mathrm{mV})$ transmembrane potentials and altered concentrations of monovalent ions such as sodium, potassium and chloride [27] [63] [64]. For example, a significant rise in intracellular sodium in highly differentiated neurons has been associated with the initiation of DNA synthesis [63]. This is particularly significant since cell electric potential and sodium ion concentration have also been associated with contact inhibition in monolayer cell cultures [65].

Primitive cells had to divide with very few biological mechanisms in place. It is therefore doubtful that their membranes had the requisite carrier molecules needed for active transport. Thus, it will be assumed here that the earliest dividing cells did not possess ion pumps. It is further assumed that a reasonable approximation for the transmembrane potential may be obtained by considering the monovalent ions $\mathrm{K}^{+}, \mathrm{Na}^{+}$and $\mathrm{Cl}^{-}$along with experimental values for the intracellular concentrations of impermeant ions. This assumption is revisited below in light of experimental data on actual cell membrane potentials.

It is reasonable to assume that repeated cell division was not only a necessity but also natural for the first successful primitive cells, and it was only later that systems of cells, such as those found in multicellular organisms, evolved "social controls" over cell division. It has been suggested that a loss of such controls is at least partially responsible for the neoplastic transformation. The loss of social control has a simple interpretation in the context of the present work regarding electrostatic considerations.

Following Benedek and Villars [66], we calculate the intracellular concentrations of sodium, potassium and chloride ions in pure passive electrodiffusive equilibrium, as it is assumed here to have been the situation for individual, isolated primitive cells whose existence depended on continuing cell division with no social controls. Typical experimental values of extracellular concentrations of potassium, sodium and chloride ions are 10, 140 and $150 \mathrm{mM}$, respectively. These values, along with an intracellular nonpermeant ion concentration $C_{n p}$ of $125 \mathrm{mM}$ [66] may be substituted into 


$$
C_{K(i)} / C_{K(o)}=C_{N a(i)} / C_{N a(o)} \text { and } C_{N a(i)} / C_{N a(o)}=C_{C l(o)} / C_{C l(i)}
$$

satisfying the requirement of overall intracellular electrical neutrality

$$
C_{K(i)}+C_{N a(i)}=C_{C l(i)}+C_{n p}
$$

to obtain intracellular concentrations of potassium, sodium and chloride ions of 15,210 and $100 \mathrm{mM}$, respectively. The transmembrane potential is then given from (6) as $-11 \mathrm{mV}$ at $T=310 \mathrm{~K}$ (body temperature). Such values for the cell potential and ionic concentrations are typical of present-day mitotically active cells.

The above calculation will be repeated with the inclusion of active pumping of sodium ions from the cell to the extracellular medium. Experimentally, the active transport of sodium ions results in an intracellular sodium concentration of $12 \mathrm{mM}$. Using this value for the intracellular sodium ion concentration, along with the same values for the extracellular concentrations of sodium, potassium and chloride ions from the previous calculation, and the same intracellular concentration $C_{n p}$ of impermeant ions, application of the equality of the Nernst potentials (6) for potassium and chloride ions, and electrical neutrality (9) to all three ions, yields $125 \mathrm{mM}$ and $12 \mathrm{mM}$ for the intracellular concentrations of potassium and chloride ions, respectively. These values satisfy the condition:

$$
C_{K(i)} / C_{K(o)}=C_{C l(o)} / C_{C l(i)}=12.5
$$

as well as intracellular electroneutrality. Since electrodiffusive equilibrium applies to potassium and chloride ions, the Nernst potentials of these ions are equal:

$$
V_{N}\left(\mathrm{~K}^{+}\right)=V_{N}\left(\mathrm{Cl}^{-}\right)
$$

Thus we have:

$$
(-k T / Z e) \ln \left(C_{K(i)} / C_{K(o)}\right)=(k T / Z e) \ln \left(C_{C l(i)} / C_{C l(o)}\right)
$$

And from the equality of concentration ratios given in (10), we have $V_{N}\left(\mathrm{~K}^{+}\right)=$ $V_{N}\left(\mathrm{Cl}^{-}\right)=-67 \mathrm{mV}$ at $\mathrm{T}=310 \mathrm{~K}$. This transmembrane potential value compares favorably to experimental values for non-dividing cells [63].

The cytosolic and nucleoplasmic ionic strength

$$
I=(1 / 2) \sum M_{n} Z_{n}^{2}
$$

where $M_{n}$ is the molal concentration, and $Z_{n}$ the ionic valence, will also be permanently different for cancer cells. This important difference will be considered below in the context of DNA replication.

\section{Cell Membrane Charge, Intramembrane Potential, Ion Concentrations and Cancer}

The calculation above reveals that a significantly smaller transmembrane potential (as observed in cancerous cells; [27] [28]) is consistent with an impaired, or absent, plasma membrane sodium pump in cycling cells. In accord with experiment [63], the calculation is in agreement with increased sodium ion 
intracellular concentration for malignant cells. Note that the observed temporary drop in transmembrane potential during mitosis for many normal cell types is not being addressed here (but see below).

Primitive biological cells likely lacked the intramembrane carrier molecules needed for active sodium export from cells (e.g., $\mathrm{Na}^{+} / \mathrm{K}^{+}$-ATPase pumps), thus it is reasonable to assume that the natural and necessary tendency for the uncontrolled cell division of ancient, unicellular organisms occurred within conditions of passive electrodiffusive equilibrium without active pumping. With the arrival of photosynthesis, planetary oxygen and multicellular organisms, the need for social controls over cell division likely required the evolution of $\mathrm{Na}^{+} / \mathrm{K}^{+}$plasma membrane pumps consistent with the large (negative) transmembrane electric potentials observed in non-cycling cells, along with the above calculated, and experimentally confirmed, ion concentrations characteristic of such cells. In multicellular organisms, these ionic concentrations are shared among large numbers of cells due to normally functioning gap junctions. A large volume of cells freely sharing non-mitogenic concentrations of ions is integral to an organism's social control over cell division, and thus increased cell surface negative charge likely compromises proper gap junction formation and the consequent maintenance of non-mitogenic ionic concentrations in multicellular organisms.

In modern cells, the carrier molecules necessary for the active transport of sodium reside within the cell membrane, and therefore sense only the intramembrane potential portion of the transmembrane electric potential [67]. From (5), the transmembrane potential $\Delta V_{m}$ is the sum of the surface potentials at the outer and inner surfaces and the intramembrane voltage $V_{d}$. This equation is key to understanding a possible role for increased negative charge at the cell surface in cancer cell mitogenesis.

Since an increased density of negatively charged sialic acid residues $\sigma$ at the outer cell membrane of cancer cells will produce a greater negative surface electric potential $\varphi_{o}$, it follows from (5) that for a given measured $\Delta V_{m}$ there must be a corresponding decrease in the magnitude (smaller negative value) or a positive value for the intramembrane potential $V_{d}$, relative to normal values [67] [68]. Measurements of transmembrane potentials are unable to resolve the potentials $\varphi_{o}$ and $\varphi_{i}$, and reflect only the total potential drop across the membrane, $\Delta V_{m}$ (Figure 2), which is due solely to the bulk concentrations of ions in the extracellular environment and in the cytosol. This is a consequence of the Boltzmann energy distribution function of classical statistical mechanics, which governs the dependence of the total potential change (the transmembrane potential) between bulk medium extracellular and intracellular ion concentrations under electrodiffusive equilibrium conditions. In addition, given that the transmembrane potential is permanently lower in magnitude (less negative) for cancerous cells, it follows that the intramembrane potential is likely positive, zero or slightly negative. This combination of conditions give sufficient basis to assume that the carrier molecules responsible for active transport of sodium ions out of a cell (ex- 
port) will sense a significantly altered intramembrane potential-as well as increased membrane electrical stress-likely affecting active sodium ion export as well as the operation of ion channels [67]. This modification of the plasma cell membrane ion transport properties of cancer cells likely has important consequences for mitogenesis (see below).

An increasing number of studies suggest that ion channels and pumps are important players in cell proliferation [69]. As demonstrated in the above calculation, the reduced transmembrane potential characteristic of cancer cells is consistent with an increased intracellular sodium ion concentration resulting from impaired-or absent-plasma membrane sodium/potassium $\left(\mathrm{Na}^{+} / \mathrm{K}^{+}\right.$-ATPase $)$ active pumping. Note that several studies show that $\left[\mathrm{Na}^{+}\right]$is significantly elevated in cancer cells [27] [70].

Experiments have revealed that the transient movement of sodium ions into cells is associated with a rise of intracellular $\mathrm{pH}\left(\mathrm{pH}_{\mathrm{i}}\right)$ that occurs when many cell types leave division arrest [71], and that a large drop in transmembrane potential occurs as cells enter mitosis [27]. It is therefore reasonable to assume that a sustained, increased intracellular sodium ion concentration, as well as a sustained lower transmembrane potential (as observed in mitotically active and cancer cells) would cause a permanently elevated $\mathrm{pH}_{\mathrm{i}}$ due to $\mathrm{Na}^{+} / \mathrm{H}^{+}$exchange [71] [72] [73] and the electrostatic requirement of intracellular electrical neutrality. Significantly, observations have revealed that cytoplasmic and nuclear $\mathrm{pH}$ are equivalent in HeLa cells [74], a well-known line of cancer cells. Alkaline pH appears to be a necessary preparatory condition for DNA synthesis [75], and studies have shown that intracellular acidification can diminish tumor growth and provoke cytotoxic death [76].

The conditions described here-1) low transmembrane potential, 2) impaired or absent $\mathrm{Na}^{+} / \mathrm{K}^{+}$plasma membrane pumps, 3) high intracellular sodium ion concentration, and 4) alkaline cellular $\mathrm{pH}_{\mathrm{i}}$ - are consistent with the reversion of cells to a more primitive mitotically active, cancerous lifestyle. In accord with experiment, the calculations above directly support the first three of these conditions, and given a high intracellular sodium ion concentration, condition (4) follows due to $\mathrm{H}^{+} / \mathrm{Na}^{+}$exchange [71] and overall electrical neutrality.

As alluded to above, the commonly observed transient influx of sodium ions and the temporary drop in transmembrane potential during normal cell division is not to be confused with the permanently lowered mitogenic intramembrane potential-with consequent failure of sodium ion active pumping-and permanently increased $\left[\mathrm{Na}^{+}\right]$discussed here. We propose that this observation is consistent with the present discussion as follows. During mitosis, the mitotic apparatus occupies most of the volume of the cell, and mitochondrial, as well as other inner membranes, disassemble into membrane fragments, likely due to increased electrostatic stress during mitosis. A full analysis of this process for the membranes of the nuclear envelope is given elsewhere [2]. Thus it seems reasonable that mitochondrial functioning to provide the ATP necessary for active pumping 
is not operative during mitosis. Consequently, the absence of $\mathrm{Na}^{+} \mathrm{K}^{+}$pumping leads to electrodiffusive equilibrium conditions, including observations of low transmembrane potential and the movement of sodium ions into cells.

It is well-known that ionic strength is significant in the biochemical reactions within polyelectrolytes like the cytoplasm of biological cells. Ionic strength calculations, based on ion concentrations in cancer cells as compared to normal counterparts, reveal that the cytosolic and nucleoplasmic ionic strengths of cancerous cells are considerably higher. Data from Cameron et al. [70] reveals that the cytoplasmic ratio of ionic strength for cancer cells relative to their normal counterparts is 1.6. Ionic strength relates to the degree of shielding of electrostatic interactions in an ionic medium. Thus it seems that the greater cytoplasmic and nucleoplasmic ionic strengths of cancer cells are significant for the protein biochemistry of these cells.

Experiments reveal that the optimum $\mathrm{pH}$ for DNA synthesis is a strong function of ionic strength [77] [78], likely due to the dependence of hydrogen bond strength on the amount of electrostatic shielding. For a polycationic environment surrounding an enzyme, the $\mathrm{pH}$ optimum will be shifted toward more acidic values; a shift toward more basic values occurs in a polyanionic environment [78]. For example, experiments show that, in RNA systems (with similar results expected for DNA systems) a polycationic ionic strength ratio of 1.5 results in an optimum $\mathrm{pH}$ shift from 8.3 to 7.5 [78]. As noted, the cytosolic ratio of ionic strengths for cancer cells relative to non-cancer cells is 1.6, with an expectation that nucleoplasmic ratios should be commensurate with this ratio. Thus it is reasonable to expect significant alterations in the DNA biochemistry of cancer cells, causing DNA synthesis to occur when it should not.

It is generally thought that once DNA replication is initiated, a cell is committed to divide. However not much is offered in the biological literature to explain the link between replication and the events of mitosis (nuclear division). Here we propose that the sudden "explosion" in the demand for protons needed for DNA replication (i.e., the rapid conversion of uracil to thymidine via the thymidylate synthase salvage pathway during $S$ phase [79]) soon depletes nuclear proton stores, causing a "proton-poor" nucleoplasm. The subsequent increased local $\mathrm{pH}$ in the extranuclear environment, and consequent increase in nuclear membrane electrostatic stress (ES), leads to fragmentation of the nuclear envelope membranes [2]. The further spread [80] of the proton-poor condition leads to the cytosolic alkalinization observed during early prophase [81] [82]; subsequently, a steadily decreasing $\mathrm{pH}_{\mathrm{i}}$ during mitosis acts as a master clock for the timing of chromosome motions during mitosis [83] [84].

\section{Conclusions}

Increased cell surface negative charge-membrane-bound, ionized carboxyl groups of sialic acid residues-is directly correlated with a number of characteristics of cancer cells. The present work relates to increased cell surface negative 
charge to aspects of mitogenesis in cancer cells including increased membrane electrostatic stress (via sialic acid) and reduced intramembrane electric potential. Both are associated with altered cytoplasmic and nucleoplasmic ionic concentrations likely integral to mitogenesis. As supported by the experiment as well as calculation, the loss of active $\mathrm{Na}^{+} / \mathrm{K}^{+}$plasma membrane pumping is consistent with increased concentrations of sodium and other ions, low trans- and intramembrane electric potential, and alkaline cytoplasmic $\mathrm{pH}$, all related to mitogenesis. Increased ionic concentrations, with attendant increased ionic strength, may result in modifications in electrostatic interactions of proteins (e.g., cell cycle checkpoints) and nucleic acids through altered charge screening, leading to conditions favoring DNA synthesis and subsequent aberrant cell division.

Abnormal cells exhibit greatly increased intercellular electrical resistance, a condition that is consistent with increased surface charge and the consequent observed electrical isolation of malignant cells. This negates the power of large cell assemblies to equalize their ionic concentrations (social control) through normally functioning gap junctions, thus allowing ionic concentrations with mitogenic potential. A failure of ionic continuity at the peripheries of abnormal, electrically isolated cells with mitogenic intracellular ionic concentrations would allow cancerous cells to grow within normal surrounding tissue (a situation not unlike the conditions for isolated unicellular organisms), a common observation in the histology of tumors.

\section{Ethics Approval and Consent to Participate}

Not applicable.

\section{Consent for Publication}

Not applicable.

\section{Availability of Data and Materials}

Not applicable.

\section{Funding}

Busch Biomedical grant to DHS.

\section{Authors' Contributions}

LJG conceptualized the theoretical aspects of this article and DHS provided intellectual contributions. Both authors read and approved the final manuscript.

\section{Acknowledgments}

Not applicable.

\section{Conflicts of Interest}

The authors declare no conflicts of interest regarding the publication of this paper. 


\section{References}

[1] Gagliardi, L.J. (2005) Electrostatic Force Generation in Chromosome Motions during Mitosis. Journal of Electrostatics, 63, 309-327. https://doi.org/10.1016/j.elstat.2004.09.007

[2] Gagliardi, L.J. (2006) Electrostatic Considerations in Nuclear Envelope Breakdown and Reassembly. Journal of Electrostatics, 64, 843-849.

https://doi.org/10.1016/j.elstat.2006.02.005

[3] Gagliardi, L.J. and Shain, D.H. (2014) Polar Electrostatic Forces Drive Poleward Chromosome Motions. Cell Division, 9, 5. https://doi.org/10.1186/s13008-014-0005-3

[4] Gagliardi, L.J. and Shain, D.H. (2014) Chromosome Congression Explained by Nanoscale Electrostatics. Theoretical Biology and Medical Modelling, 11, Article No. 12. https://doi.org/10.1186/1742-4682-11-12

[5] Gagliardi, L.J. and Shain, D.H. (2016) Electrostatic Forces Drive Poleward Chromosome Motions at Kinetochores. Cell Division, 11, 14. https://doi.org/10.1186/s13008-016-0026-1

[6] van Beek, W.P., Smets, L.A. and Emmelot, P. (1973) Increased Sialic Acid Density in Surface Glycoprotein of Transformed and Malignant Cells-A General Phenomenon? Cancer Research, 33, 2913-2922.

[7] Seaman, G.V.F. and Cook, G.M.W. (1965) Modification of the Electrophoretic Behaviour of the Erythrocyte by Chemical and Enzymatic Methods. In: Ambrose, E.J., Ed., Cell Electrophoresis, J \& A Churchill Ltd., London, 48-65.

[8] Sand, S.L., Nissen-Meyer, J., Sand, O. and Haug, T.M. (2013) Plantaricin A, a Cationic Peptide Produced by Lactobacillus plantarum, Permeabilizes Eukaryotic Cell Membranes by a Mechanism Dependent on Negative Surface Charge Linked to Glycosylated Membrane Proteins. Biochimica et Biophysica Acta Biomembranes, 1828, 249-259. https://doi.org/10.1016/j.bbamem.2012.11.001

[9] Du, J., Meledeo, M.A., Wang, Z., Kanna, H.S., Paruchuri, V.D.P. and Yarema, K.J. (2009) Metabolic Glycoengineering: Sialic Acid and Beyond. Glycobiology, 19, 1382-1401. https://doi.org/10.1093/glycob/cwp115

[10] Tamura, A., Morita, K., Fujii, T. and Kojima, K. (1982) Detection of the Electrical Surface Charge Induced by Treatment of the Membrane Lipid Bilayer of Human Erythrocytes. Cell Structure and Function, 7, 21-27. https://doi.org/10.1247/csf.7.21

[11] Corfield, A. (2017) Eukaryotic Protein Glycosylation: A Primer for Histochemists and Cell Biologists. Histochemistry and Cell Biology, 147, 119-147. https://doi.org/10.1007/s00418-016-1526-4

[12] Weiss, L. and Mayhew, E. (1969) Ribonuclease-susceptible Charged Groups at the Surface of Ehrlich Ascites Tumour Cells. International Journal of Cancer, 4, 626-635. https://doi.org/10.1002/ijc.2910040507

[13] Wolstenholme, G.E.W. and Knight, J. (1971) Growth Control in Cell Cultures. Churchill Livingstone, Edinburgh.

[14] Buck, C.A., Glick, M.C. and Warren, L. (1971) Glycopeptides from the Surface of Control and Virus-Transformed Cells. Science, 172, 169-171. https://doi.org/10.1126/science.172.3979.169

[15] Warren, L., Fuhrer, J.P. and Buck, C.A. (1972) Surface Glycoproteins of Normal and Transformed Cells: A Difference Determined by Sialic Acid and a Growth-Dependent Sialyl Transferase. Proceedings of the National Academy of Sciences, 69, 1838-1842. 
https://doi.org/10.1073/pnas.69.7.1838

[16] Loewenstein, W.R. and Kanno, Y. (1966) Intercellular Communication and the Control of Tissue Growth: Lack of Communication between Cancer Cells. Nature, 209, 1248-1249. https://doi.org/10.1038/2091248a0

[17] Fedarko, N.S., Ishihara, M. and Conrad, H.E. (1989) Control of Cell Division in Hepatoma Cells by Exogenous Heparan Sulfate Proteoglycan. Journal of Cellular Physiology, 139, 287-294. https://doi.org/10.1002/jcp.1041390210

[18] Baserga, R. (1965) The Relationship of the Cell Cycle to Tumor Growth and Control of Cell Division: A Review. Cancer Research, 25, 581-595.

[19] Cormack, D. (1970) Effect of Enzymatic Removal of Cell Surface Sialic Acid on the Adherence of Walker 256 Tumor Cells to Mesothelial Membrane. Cancer Research, 30, 1459-1466.

[20] Bassaganas, M., Perez-Garay, M. and Peracaula, R. (2012) Adhesive and Migratory Behaviour of Pancreatic Adenocarcinoma Capan-1 Cells Is Regulated by Changes in Sialic Acid of Alpha2beta1 Integrin. European Journal of Cancer, 48, S57. https://doi.org/10.1016/S0959-8049(12)70927-9

[21] Bassaganas, S., Perez-Garay, M. and Peracaula, R. (2014) Cell Surface Sialic Acid Modulates Extracellular Matrix Adhesion and Migration in Pancreatic Adenocarcinoma Cells. Pancreas, 43, 109-117. https://doi.org/10.1097/MPA.0b013e31829d9090

[22] Shanmugam, V., Chackalaparampil, I., Kundu, G.C., Mukherjee, A. and Mukherjee, B.B. (1997) Altered Sialylation of Osteopontin Prevents Its Receptor-Mediated Binding on the Surface of Oncogenically Transformed tsB77 Cells. Biochemistry, 36, 5729-5738. https://doi.org/10.1021/bi961687w

[23] Gasic, G. and Gasic, T. (1962) Removal of Sialic Acid from the Cell Coat in Tumor Cells and Vascular Endothelium, and Its Effects on Metastasis. Proceedings of the National Academy of Sciences of the United States of America, 48, 1172-1177. https://doi.org/10.1073/pnas.48.7.1172

[24] Kemp, R.B. (1968) Effect of the Removal of Cell Surface Sialic Acids on Cell Aggregation in Vitro. Nature, 218, 1255-1256. https://doi.org/10.1038/2181255a0

[25] Benedetti, E.L. and Emmelot, P. (1976) Studies on Plasma Membranes. Journal of Cell Science, 2, 499-512.

[26] Roth, S. and White, D. (1972) Intercellular Contact and Cell-Surface Galactosyl Transferase Activity. Proceedings of the National Academy of Sciences of the United States, 69, 485-489. https://doi.org/10.1073/pnas.69.2.485

[27] Cone, C.D. (1971) Unified Theory on the Basic Mechanism of Normal Mitotic Control and Oncogenesis. Journal of Theoretical Biology, 30, 151-181. https://doi.org/10.1016/0022-5193(71)90042-7

[28] Cone, C.D. (1971) Maintenance of Mitotic Homeostasis in Somatic Cell Populations. Journal of Theoretical Biology, 30, 183-194. https://doi.org/10.1016/0022-5193(71)90043-9

[29] Levin, M. (2012) Molecular Bioelectricity in Developmental Biology: New Tools and Recent Discoveries. Bioessays, 34, 205-217. https://doi.org/10.1002/bies.201100136

[30] Gurtovenko, A.A. and Vattulainen, I. (2009) Intrinsic Potential of Cell Membranes: Opposite Effects of Lipid Transmembrane Asymmetry and Asymmetric Salt Ion Distribution. Journal of Physical Chemistry B, 113, 7194-7198. https://doi.org/10.1021/jp902794q

[31] Shaefer, H. and Schanne, O. (1956) Membranpotentiale von Einzelzellen in Gewe- 
bekulturen. Naturwissenschafften, 43, 445. https://doi.org/10.1007/BF00629508

[32] Tokuoka, S. and Morioka, H. (1957) The Membrane Potential of the Human Cancer and Related Cells. Gann, 48, 353-354.

[33] Johnstone, B.M. (1959) Micro-Electrode Penetration of Ascites Tumour Cells. Nature, 183, 411. https://doi.org/10.1038/183411a0

[34] Griffiths, D.J. (1999) Introduction to Electrodynamics. Prentice-Hall, Upper Saddle River, 103.

[35] Weiss, L. (1968) Studies on Cell Deformability: V. Some Effects of Ribonuclease. Journal of Theoretical Biology, 18, 9-18. https://doi.org/10.1016/0022-5193(68)90167-7

[36] Bockris, J.O. and Reddy, A.K.N. (1977) Modern Electrochemistry. Plenum Press, New York.

[37] Teschke, O., Ceotto, G. and de Souza, E.F. (2001) Interfacial Water Dielectric-Permittivity Profile Measurements Using Atomic Force Microscopy. Physical Review E, 64, Article ID: 011605. https://doi.org/10.1103/PhysRevE.64.011605

[38] Pashley, R.M. (1981) DLVO and Hydration Forces between Mica Surfaces in $\mathrm{Li}^{+}$, $\mathrm{Na}^{+}, \mathrm{K}^{+}$and $\mathrm{Cs}^{+}$Electrolyte Solutions: A Correlation of Double-Layer and Hydration Forces with Surface Cation Exchange Properties. Journal of Colloid and Interface Science, 83, 531-546. https://doi.org/10.1016/0021-9797(81)90348-9

[39] Heinz, W.F. and Hoh, J.H. (1999) Relative Surface Charge Density Mapping with the Atomic Force Microscope. Biophysical Journal, 76, 528-538. https://doi.org/10.1016/S0006-3495(99)77221-8

[40] Giese, A.C. (1968) Cell Physiology. W.B. Saunders, Philadelphia, 104.

[41] Segal, J.R. (1968) Surface Charge of Giant Axons of Squid and Lobster. Biophysical Journal, 8, 470-489. https://doi.org/10.1016/S0006-3495(68)86501-4

[42] Fettiplace, R., Andrews, D.M. and Haydon, D.A. (1971) The Thickness, Composition and Structure of Some Lipid Bilayers and Natural Membranes. Journal of Membrane Biology, 5, 277-296. https://doi.org/10.1007/BF01870555

[43] Mayhew, E. (1966) Cellular Electrophoretic Mobility and the Mitotic Cycle. The Journal of General Physiology, 49, 717-725. https://doi.org/10.1085/jgp.49.4.717

[44] Mayhew, E. and O'Grady, E.A. (1965) Electrophoretic Mobilities of Tissue Culture Cells in Exponential and Parasynchronous Growth. Nature, 207, 86-87. https://doi.org/10.1038/207086a0

[45] Glick, M.C., Gerner, E.W. and Warren, L. (1971) Changes in the Carbohydrate Content of the KB Cell during the Growth Cycle. Journal of Cellular Physiology, 77, 1-5. https://doi.org/10.1002/jcp.1040770102

[46] Anderson, A.P., Moreira, J.M.A. and Pedersen, S.F. (2014) Interactions of Ion Transporters and Channels with Cancer Cell Metabolism and the Tumour Environment. Philosophical Transactions of the Royal Society B, 369, Article ID: 20130098. https://doi.org/10.1098/rstb.2013.0098

[47] Alberts, B., Bray, D., Lewis, J., Raff, M., Roberts, K. and Watson, J.D. (1994) Molecular Biology of the Cell. 3rd Edition, Garland Publishing, New York, 1077.

[48] Kemp, R.B. (1970) The Effect of Neuraminidase (3:2:1:18) on the Aggregation of Cells Dissociated from Embryonic Chick Muscle Tissue. Journal of Cell Science, 6, 751-766.

[49] Morell, A.G., Gregoriades, G., Scheinberg, I.H., Hickman, J. and Ashwell, G. (1971) The Role of Sialic Acid in Determining the Survival of Glycoproteins in the Circula- 
tion. Journal of Biological Chemistry, 246, 1461-1467.

[50] Woodruff, J.J. and Gesner, B.B. (1969) The Effect of Neuraminidase on the Fate of Transfused Lymphocytes. Journal of Experimental Medicine, 129, 551-567. https://doi.org/10.1084/jem.129.3.551

[51] Culp, L.A., Grimes, W.J. and Black, P.H. (1971) Contact Inhibited Revertant Cell Lines Isolated from SV40-Transformed Cells. Journal of Cell Biology, 50, 682-690. https://doi.org/10.1083/jcb.50.3.682

[52] Perdue, J.F., Kletzien, R. and Wray, V.L. (1972) The Isolation and Characterization of Plasma Membrane from Cultured Cells: IV. The Carbohydrate Composition of Membranes Isolated from Oncogenic RNA Virus-Converted Chick Embryo Fibroblasts. Biochimica et Biophysica Acta Biomembranes, 266, 505-510. https://doi.org/10.1016/0005-2736(72)90106-X

[53] Vaheri, A., Ruoslahti, E. and Nordling, S. (1972) Neuraminidase Stimulates Division and Sugar Uptake in Density-Inhibited Cell Cultures. Nature New Biology, 238, 211-212. https://doi.org/10.1038/newbio238211a0

[54] Goldman, D.E. (1943) Potential, Impedance, and Rectification in Membranes. Journal of General Physiology, 27, 37-60. https://doi.org/10.1085/igp.27.1.37

[55] Hodgkin, A.L. and Katz, B. (1949) The Effect of Sodium Ions on the Electrical Activity of the Giant Axon of the Squid. Journal of Physiology, 108, 37-77. https://doi.org/10.1113/jphysiol.1949.sp004310

[56] Ohki, S. (1981) Membrane Potential, Surface Potential, and Ionic Permeabilities. Physiological Chemistry and Physics, 13, 195-210.

[57] Ohki, S. (1971) Electrical Potential of an Asymmetric Membrane. Journal of Colloid and Interface Science, 37, 318-324. https://doi.org/10.1016/0021-9797(71)90299-2

[58] Ohki, S. (1972) Membrane Potential of Phospholipid Bilayers: Ion Concentration and $\mathrm{pH}$ Difference. Biomembranes, 282, 55-71. https://doi.org/10.1016/0005-2736(72)90310-0

[59] MacDonald, R.C. and Bangham, A.D. (1972) Comparison of Double Layer Potentials in Lipid Monolayers and Lipid Bilayer Membranes. Journal of Membrane Biology, 7, 29-53. https://doi.org/10.1007/BF01867908

[60] Hille, B., Woodhull, A.M. and Shapiro, B.I. (1975) Negative Surface Charge near Sodium Channels of Nerve: Divalent Ions, Monovalent Ions, and pH. Philosophical Transactions of the Royal Society of London. Series B, Biological Sciences, 270, 301-318. https://doi.org/10.1098/rstb.1975.0011

[61] Hille, B. (2001) Ion Channels of Excitable Membranes. Sinauer, Sunderland, 656.

[62] Hille, B. (2001) Ion Channels of Excitable Membranes. Sinauer, Sunderland, 15.

[63] Stillwell, E.F., Cone, C.M. and Cone, C.D. (1973) Stimulation of DNA Synthesis in CNS Neurones by Sustained Depolarisation. Nature New Biology, 246, 110-111. https://doi.org/10.1038/newbio246110a0

[64] McDonald, T.F., Sachs, H.G., Orr, C.W. and Ebert, J.D. (1972) External Potassium and Baby Hamster Kidney Cells: Intracellular Ions, ATP, Growth, DNA Synthesis and Membrane Potential. Developmental Biology, 28, 290-303.

https://doi.org/10.1016/0012-1606(72)90145-5

[65] Cone, C.D. and Tongier, M. (1973) Contact Inhibition of Division: Involvement of the Electrical Transmembrane Potential. Journal of Cellular Physiology, 82, 373-386. https://doi.org/10.1002/jcp.1040820307

[66] Benedek, G.B. and Villars, F.M.H. (2000) Physics: With Illustrative Examples from Medicine and Biology: Electricity and Magnetism. Springer Verlag, New York, 478. 
https://doi.org/10.1007/978-1-4612-1228-7

[67] Hille, B. (2001) Ion Channels of Excitable Membranes. Sinauer, Sunderland, 646-657.

[68] Van der Kloot, W.G. and Cohen, I. (1979) Membrane Surface Potential Changes May Alter Drug Interactions: An Example, Acetylcholine and Curare. Science, 203, 1351-1352. https://doi.org/10.1126/science.424757

[69] Litan, A. and Langhans, S. (2015) Cancer as a Channelopathy: Ion Channels and Pumps in Tumor Development and Progression. Frontiers in Cellular Neuroscience, 9, 86. https://doi.org/10.3389/fncel.2015.00086

[70] Cameron, I.L., Smith, K.R. and Skehan, P. (1982) Energy Dispersive Spectroscopy in the Study of the Ionic Regulation of Growth in Normal and Tumor Cells. Proceedings of the Symposium on Ions, Cell Proliferation, and Cancer, Essex, 13-40. https://doi.org/10.1016/B978-0-12-123050-0.50007-2

[71] Wolfe, S.L. (1993) Molecular and Cellular Biology. 2nd Edition, Wadsworth, Belmont, 920 .

[72] Alberts, B., Bray, D., Hopkin, K., Johnson, A., Lewis, J., Raff, M., Roberts, K. and Walter, P. (2010) Essential Cell Biology. Third Edition, Garland Science, New York, 401.

[73] Moolenaar, W.H., de Laat, S.W., Mummery, C. and van der Saag, P.T. (1982) $\mathrm{Na}^{+} / \mathrm{H}^{+}$Exchange in the Action of Growth Factors. Proceedings of the Symposium on Ions, Cell Proliferation, and Cancer, Essex, 151-162. https://doi.org/10.1016/B978-0-12-123050-0.50015-1

[74] Selsek, O. and Bolard, J. (1996) Nuclear pH Gradient in Mammalian Cells Revealed by Microspectrofluorimetry. Journal of Cell Science, 109, 257-262.

[75] McBrian, M.A., Behbahan, I.S., Ferrari, R., Su, T., Huang, T., Li, K., Hong, C.S., Christofk, H.R., Vogelauer, M., Seligson, D.B. and Kurdistani, S.K. (2013) Histone Acetylation Regulates Intracellular pH. Molecular Cell, 49, 310-321. https://doi.org/10.1016/j.molcel.2012.10.025

[76] Garcia-Canero, R., Trilla, C., Perez de Diego, Diaz-Fil, J.J. and Cobo, J.M. (1999) $\mathrm{Na}^{+}: \mathrm{H}^{+}$Exchange Inhibition Induces Intracellular Acidosis and Differentially Impairs Cell Growth and Viability of Human and Rat Hepatocarcinoma Cells. Toxicology Letters, 106, 215-228. https://doi.org/10.1016/S0378-4274(99)00072-7

[77] Byrnes, J.J., Downey, K.M. and So, A.G. (1973) Bone Marrow Cytoplasmic Deoxyribonucleic Acid Polymerase. Variation of $\mathrm{pH}$ and Ionic Environment as a Possible Control Mechanism. Biochemistry, 12, 4378-4384. https://doi.org/10.1021/bi00746a013

[78] Maurel, P. and Douzou, P. (1978) Cation-Induced Regulatory Mechanism of Enzyme Reactions. In: Frontiers in Physicochemical Biology, Academic Press, New York, 421-457. https://doi.org/10.1016/B978-0-12-566960-3.50024-5

[79] Berg, J.M., Tymoczko, J.L. and Stryer, L. (2011) Biochemistry. 7th Edition, Palgrave Macmillan, London, 1026.

[80] Gagliardi, L.J. (1973) A Possible Mechanism for Protonic Transfer in Aqueous Solutions. Journal of Physical Chemistry, 77, 2098-2100. https://doi.org/10.1021/j100636a014

[81] Amirand, C., et al. (2000) Intracellular pH in One Cell Mouse Embryo Differs between Subcellular Compartments and between Interphase and Mitosis. Biology of the Cell, 92, 409-419. https://doi.org/10.1016/S0248-4900(00)01080-7

[82] Steinhardt, R.A. and Morisawa, M. (1982) Changes in Intracellular pH of Physarum 
plasmodium during the Cell Cycle and in Response to Starvation. In: Nuccitelli, R. and Deamer, D.W., Eds., Intracellular pH: Its Measurement, Regulation, and Utilization in Cellular Functions, Alan R. Liss, New York, 361-374.

[83] Gagliardi, L.J. and Shain, D.H. (2013) Is Intracellular pH a Clock for Mitosis? Theoretical Biology and Medical Modelling, 10, 8.

https://doi.org/10.1186/1742-4682-10-8

[84] Gagliardi, L.J. and Shain, D.H. (2018) Emergent Mitotic Chromosome Motions from a Changing Intracellular pH. Open Journal of Biophysics, 8, 9-21.

https://doi.org/10.4236/ojbiphy.2018.81002 